\title{
Aluminum abundances of multiple stellar generations in the globular cluster NGC $1851^{\star, \star \star}$ (Research Note)
}

\author{
E. Carretta ${ }^{1}$, V. D’Orazi ${ }^{2}$, R. G. Gratton ${ }^{3}$, and S. Lucatello ${ }^{3}$
}

\author{
1 INAF - Osservatorio Astronomico di Bologna, via Ranzani 1, 40127 Bologna, Italy \\ e-mail: eugenio.carretta@oabo.inaf.it \\ 2 Dept. of Physics and Astronomy, Macquarie University, Sydney, NSW, 2109 Australia \\ 3 INAF - Osservatorio Astronomico di Padova, Vicolo dell'Osservatorio 5, 35122 Padova, Italy \\ Received 26 March 2012 / Accepted 30 May 2012
}

\section{ABSTRACT}

\begin{abstract}
We studied the distribution of aluminum abundances among red giants in the peculiar globular cluster NGC 1851. Aluminum abundances were derived from the strong doublet Al I 8772-8773 Å measured on intermediate-resolution FLAMES spectra of 50 cluster stars acquired under the Gaia-ESO public survey. We coupled these abundances with previously derived abundances of $\mathrm{O}$, Na, and $\mathrm{Mg}$ to fully characterize the interplay of the $\mathrm{NeNa}$ and $\mathrm{MgAl}$ cycles of $\mathrm{H}$-burning at high temperature in the early stellar generation in NGC 1851. The stars in our sample show well-defined correlations between $\mathrm{Al}, \mathrm{Na}$ and $\mathrm{Si}$; $\mathrm{Al}$ is anticorrelated with $\mathrm{O}$ and $\mathrm{Mg}$. The average value of the $[\mathrm{Al} / \mathrm{Fe}]$ ratio steadily increases from the first generation stars to the second generation populations with intermediate and extremely modified composition. We confirm on a larger database the results recently obtained by us: the pattern of abundances of proton-capture elements implies a moderate production of Al in NGC 1851. We find evidence of a statistically significant positive correlation between $\mathrm{Al}$ and $\mathrm{Ba}$ abundances in the more metal-rich component of red giants in NGC 1851.
\end{abstract}

Key words. stars: abundances - stars: atmospheres - stars: Population II - globular clusters: general -

globular clusters: individual: NGC 1851

\section{Introduction}

The ubiquitous presence in galactic globular clusters (GCs) of multiple stellar populations is amply assessed from the wealth of recent, high-quality data (see e.g. the reviews by Gratton et al. 2004; Martell 2011; Gratton et al. 2012a). Spectroscopy, in particular (but in a few cases also the splitting of photometric sequences, see e.g. Piotto 2009; Bragaglia 2010) proves beyond all doubt that the stellar population in GCs is composed of at least two stellar generations, distinct in ages (with a slight age difference from a few million years up to a few $10^{7}$ years, depending on the nature of polluters) and in particular in a chemical composition even hugely different between each other, although these two generations may not be often seen as discrete groups in the abundance planes and/or in the colour-magnitude diagram.

This chemical signature must necessarily be attributed to the action of the more massive stars of an early, first generation because only this type of stars could be responsible for the present pattern of $\mathrm{Na}-\mathrm{O}$ and $\mathrm{Mg}-\mathrm{Al}$ anticorrelations observed in presentday unevolved cluster stars (Gratton et al. 2001), through the nuclear processing of $\mathrm{H}$ burning at high temperature (Denisenkov \& Denisenkova 1989; Langer et al. 1993).

Most evidence concerning multiple stellar populations, and the recently found links with global cluster parameters (such as total mass, Carretta et al. 2010; or horizontal branch morphology, Gratton et al. 2010) mainly stems from the Na-O anticorrelation, which was extensively studied thanks to modern facilities such as FLAMES/VLT (e.g. Carretta et al. 2009a,b). This

\footnotetext{
* Based on observations collected at ESO telescopes under programme 188.B-3002.

$\star \star$ Table 1 is available in electronic form at http://www . aanda.org
}

feature was soon realized to be related to the intrinsic mechanism of GC formation (Carretta 2006) and it is so widespread among GCs that it can be considered as the simplest working definition of bona fide globular cluster (Carretta et al. 2010). The Na-O anticorrelation is found in almost all GCs were $\mathrm{Na}, \mathrm{O}$ abundances are derived for a large number of stars, although some claims - based on theoretical arguments - of single-generation clusters was made by e.g. D' Antona \& Caloi (2008). As an example they suggested that NGC 6397 was composed only of second generation stars. However, both Carretta et al. (2010, using mainly Na) and Lind et al. (2011, with both $\mathrm{Na}$ and $\mathrm{O}$ ) were able to show the presence of two stellar generations also in this globular cluster as well.

However, since the study by Gratton et al. (2001), the importance of collecting the widest range of elements produced in proton-capture reactions was immediately clear. In particular, the "heaviest" light elements involved, such as $\mathrm{Mg}, \mathrm{Al}$, and $\mathrm{Si}$, allow us to explore the hottest regime and cycles of the $\mathrm{H}$ burning. This is crucial to shed light on the still uncertain nature of the candidate polluters of the first stellar generation, whose identification appears to be still problematic and debated, with favourite candidates being fast rotating massive stars (FRMS: Decressin et al. 2007) or intermediate-mass asymptotic giant branch (AGB) stars (Ventura et al. 2001). In a few cases, like M 22 (Marino et al. 2009) and NGC 1851 (Carretta et al. 2011a), a contribution by type II supernovae (SNe) to the cluster pollution must also be taken into account.

To this aim we started to add $\mathrm{Al}$ abundances to the very large dataset with $\mathrm{O}, \mathrm{Na}, \mathrm{Mg}$, and $\mathrm{Si}$ abundances already in hand, by re-observing a large sample of stars of our FLAMES survey of GCs. Proprietary data are being analysed for a few key 
objects (see the results on NGC 6752, Carretta et al. 2012). In the present note we exploit the observations made of NGC 1851 by the Gaia-ESO spectroscopic public survey, which has just started on FLAMES/VLT.

\section{Observations}

We retrieved two exposures of $600 \mathrm{~s}$ each made with FLAMES mounted at VLT-UT2 and the high-resolution grating HR21 from the ESO archive. The observations were carried out on UT 17 February 2012, with airmass $z=1.115$ and 1.155 , respectively. The resolution of HR21 is 17300 and the spectral range is from $8484 \AA$ to $9001 \AA$, including the $\mathrm{Al}$ I doublet at $8772-73 \AA$, which is the feature we used.

Data reduction was performed through the ESO FLAMESGiraffe pipeline ${ }^{1}$, which provides bias-corrected, flat-fielded, 1D-extracted, and wavelength-calibrated spectra. Sky subtraction, combination of the two single exposures for each star, and rest-frame traslation were then carried out within $\mathrm{IRAF}^{2}$.

A total of 84 stars were observed in the two exposures, but we restricted our attention to the subsample of 63 stars in common with the database by Carretta et al. (2011a). For these stars we already have a full abundance analysis, with homogeneously determined atmospheric parameters, and abundances of light elements $\mathrm{O}, \mathrm{Na}, \mathrm{Mg}$, and $\mathrm{Si}$. The spectral range of HR21 contains no useful $\mathrm{Fe}$ lines, hence we restricted the present analysis to this subsample. It was not possible to measure reliable Al abundances for 13 stars that are too hot (effective temperature higher than about $4900 \mathrm{~K}$ ) or have only low-quality spectra. Hence the final sample with a complete set of light-element abundances is represented by 50 red giant branch (RGB) stars, in a range of two magnitudes (from $V \sim 17$ to $V \sim 15$ ) approximately centred at the luminosity of the bump on the RGB ( $V=16.16$, see Fig. 1). Relevant information on this final sample is listed in Table 1.

The cluster was observed as calibrator for the survey, therefore the desired signal-to-noise ratio $(\mathrm{S} / \mathrm{N})$ was set to the average one for survey stars, and was not optimised to obtain a $\mathrm{S} / \mathrm{N}$ also acceptable for the much weaker $\mathrm{Al}$ lines. The $\mathrm{S} / \mathrm{N}$ at $8800 \AA$, as measured on the combined spectra, range from 12 to 147 for the sample, with a median value of 57 . The average values of the $\mathrm{S} / \mathrm{N}$ in 0.5 mag bins are shown in Fig. 1, while individual values for each star are listed in Table 1.

In Fig. 2 (upper panel) we compare the spectra of two stars with very similar atmospheric parameters (effective temperature $T_{\text {eff }}$, surface gravity $\log g$, overall metal abundance $[\mathrm{Fe} / \mathrm{H}]$, and microturbulent velocity $\left.V_{\mathrm{t}}\right)$ : star $44939(4425 / 1.60 /-$ $1.15 / 1.01)$ and star $35999(4442 / 1.64 /-1.15 / 0.81)$, with $\mathrm{S} / \mathrm{N}$ of 147 and 49, respectively. These are the coolest giants in our sample. We also superimpose the synthetic spectrum computed with the atmospheric parameters appropriate for star 35999 and no $\mathrm{Al}([\mathrm{Al} / \mathrm{Fe}]=-9.99)$, to show the amount of possible contamination of $\mathrm{Al}$ lines by $\mathrm{CN}$ features (blue thin solid line). Another example is shown in the middle panel of Fig. 2, where we compare a pair of RGB stars with similar parameters (star 22360:

\footnotetext{
1 Version 2.8.9, http://www.eso.org/sci/software/ pipelines//giraffe/giraf-pipe-recipes.html

2 IRAF is the Image Reduction and Analysis Facility, a general purpose software system for the reduction and analysis of astronomical data. IRAF is written and supported by the IRAF programming group at the National Optical Astronomy Observatories (NOAO) in Tucson, Arizona. NOAO is operated by the Association of Universities for Research in Astronomy (AURA), Inc. under cooperative agreement with the National Science Foundation.
}

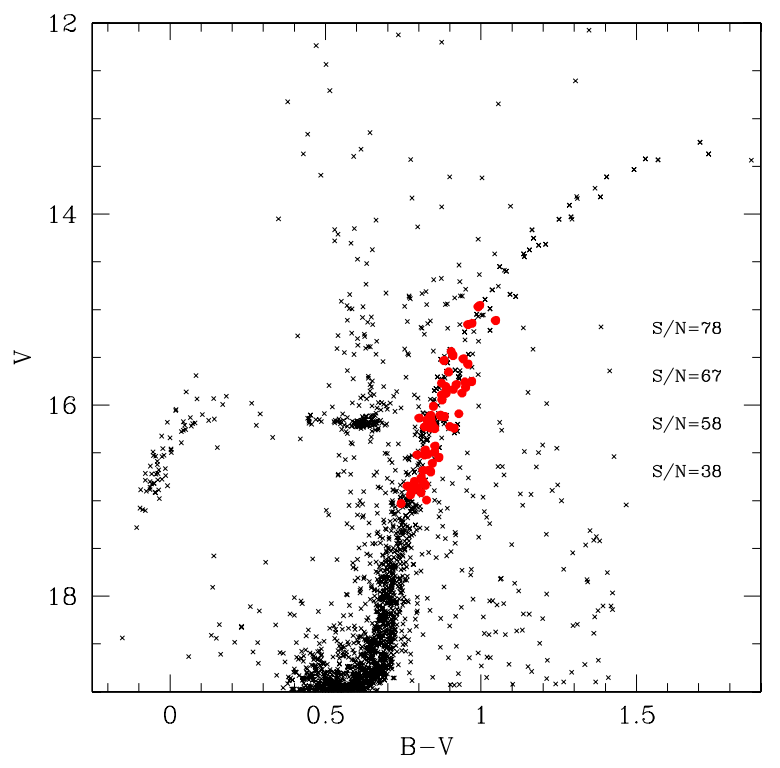

Fig. 1. $V, B-V$ colour-magnitude diagram (CMD) of NGC 1851 (grey crosses). Superimposed as large red filled circles are the giants with $\mathrm{Al}$ abundances measured in the present study. The average $\mathrm{S} / \mathrm{N}$ of spectra in bin of half a magnitude is reported to the right of the CMD.

4755/2.27/-1.15/0.86 and star 31520: 4756/2.28/-1.23/1.26) and $\mathrm{S} / \mathrm{N}$ close to the median value for the sample $(S / N=60$ and 61 for stars 22360 and 31520, respectively).

\section{Analysis}

Owing to the quality of spectra and to the weakness of Al I lines in fairly warm giants we decided to derive the abundances from the comparison of the observed flux in the region of $\mathrm{Al}$ lines with the flux measured on synthetic spectra computed using the package ROSA (Gratton 1988), with the following procedure.

First, to account for the contamination of $\mathrm{CN}$ lines, present over the spectral range where $\mathrm{Al}$ lines lie, we first reproduced a $\mathrm{CN}$ feature at $\sim 8770.5 \AA$, adopting a fixed $\mathrm{C}$ abundance $(0.0 \mathrm{dex})$ and varying the $[\mathrm{N} / \mathrm{Fe}]$ ratio until the $\mathrm{CN}$ line was reasonably well fitted. The resulting values of $[\mathrm{N} / \mathrm{Fe}]$ are listed in Table 1. The $\mathrm{C}$ content adopted in this process is very likely much higher than the actual one, which for metal-poor giants in this evolutionary stage is expected to be about -0.6 dex. However, the meaning of $[\mathrm{N} / \mathrm{Fe}]$ ratios is only indicative, since we lack precise abundances of $\mathrm{C}$, therefore these $\mathrm{N}$ abundances only indicate the value that reproduces the $\mathrm{CN}$ feature adequately. Other contaminants, such as $\mathrm{TiO}$ molecular lines, are not important.

Our line list, originally from Plez, was slightly modified by optimising the position and strength of $\mathrm{CN}$ and $\mathrm{Al}$ lines to fit the high-resolution spectrum of the cool and metal-rich ( $\mathrm{CN}$ rich) giant $\mu$ Leo (see Gratton et al. 2006).

Second, we coadded the observed spectra of the 10 stars with the highest $\mathrm{S} / \mathrm{N}$ in our sample. On this spectrum we selected a region that included the two $\mathrm{Al}$ lines and two other regions to be used to derive a local reference continuum. These regions are shown as shaded red and light blue areas in Fig. 2. We then measured the average fluxes within the in-line region $\left(f_{\mathrm{Al}}\right)$ and the reference continuum regions $\left(f_{1}\right.$ and $\left.f_{2}\right)$. We also estimated photometric errors in these regions from the $\mathrm{S} / \mathrm{N}$ of the spectra and width (and then number of pixels) within each of these regions. Finally, we defined a line strength index for the Al lines as $I_{\mathrm{Al}}=2 f_{\mathrm{Al}} /\left(f_{1}+f_{2}\right)$, with an error obtained by a 


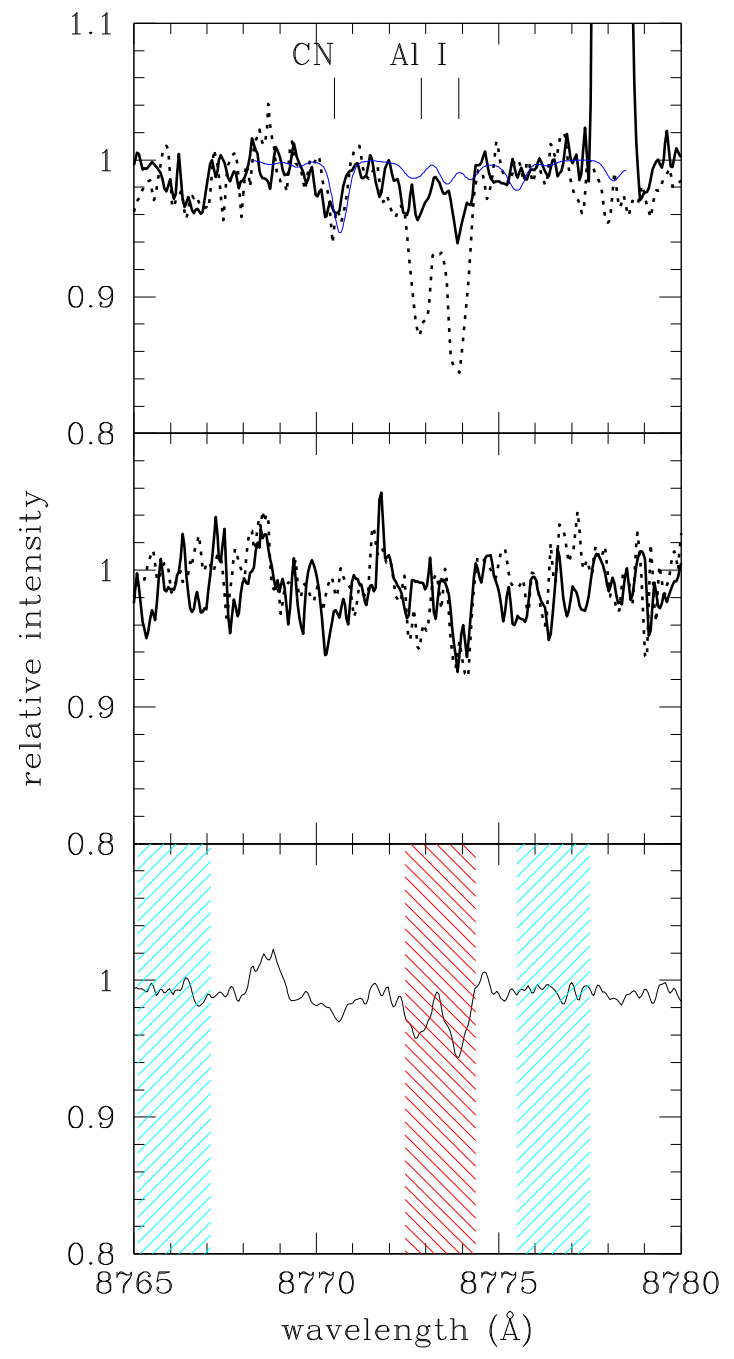

Fig. 2. Upper panel: comparison of the observed spectra for star 44939 (solid line, $[\mathrm{Al} / \mathrm{Fe}]=-0.19$ dex) and star 35999 (dotted line, $[\mathrm{Al} / \mathrm{Fe}]=+1.06 \mathrm{dex})$. The thin blue solid line is the synthetic spectrum with no $\mathrm{Al}([\mathrm{Al} / \mathrm{Fe}]=-9.99)$ computed with the atmospheric parameters relative to star 35999. Middle panel: the same for star 22360 (solid line, $[\mathrm{Al} / \mathrm{Fe}]<-0.09$ ) and star 31520 (dotted line, $[\mathrm{Al} / \mathrm{Fe}]=+0.44$ ). Lower panel: co-added spectrum of the 10 stars with highest $\mathrm{S} / \mathrm{N}$. The areas hatched in red and light blue mark the regions used to evaluate the flux appropriate for $\mathrm{Al}$ lines as discussed in the text; red is the in-line region, light blue indicates the reference for the continuum.

suitable combination of the photometric errors. The same procedure was then repeated on a set of three synthetic spectra computed for each star using the appropriate atmospheric parameters (from Carretta et al. 2011a) and abundances of $[\mathrm{Al} / \mathrm{Fe}]=-0.5$, $0.0,0.5$. Abundances of $\mathrm{Al}$ for each stars were then derived by interpolating the normalised flux in the line region among those obtained from the synthetic spectra. An error can be attached by comparing these $\mathrm{Al}$ abundances with those obtained entering a new value of the $\mathrm{Al}$ line strength index that is the sum of the original value and of its error. Whenever the measured value for the Al line strength index was lower than twice the error, we considered that only an upper limit to $\mathrm{Al}$ abundances could be obtained. In this case, we assumed that the upper limit to Al abundances be equal to three times the error: this is a quite robust estimate of the upper limit. This procedure avoids any subjective judgement, as suggested by the referee, and was applied to all the observed spectra with an $S / N>25$ : for lower $S / N$ spectra we only obtained high upper limits that do not bear important information.

We notice that typical errors associated to the $\mathrm{Al}$ abundances obtained following this approach are in the range 0.1-0.4 dex. Star-to-star errors in the adopted atmospheric parameters are quite small (see Carretta et al. 2011a) and less a source of concern. As discussed in Carretta et al. (2012), NLTE effects are not a source of concern in the star-to-star analysis in NGC 6752; this is even more true when considering more metal-rich stars, as in the present case.

\section{Results and discussion}

The derived abundances of $\mathrm{Al}$ for our sample are listed in Table 1 . We obtained $[\mathrm{Al} / \mathrm{Fe}]$ ratios for 60 stars, with 49 detection and 11 upper limits. Other abundances (for $\mathrm{Fe}, \mathrm{O}, \mathrm{Na}, \mathrm{Mg}$, Si) were taken from the analysis of Carretta et al. (2011a) and are repeated for convenience in this table. As mentioned in the introduction, they are available for 50 stars.

$\mathrm{Al}$ abundances do not present any significant trend as a function of effective temperature or metallicity $[\mathrm{Fe} / \mathrm{H}]$. The expected correlations of $\mathrm{Al}$ with elements enhanced by proton-capture reactions ( $\mathrm{Na}, \mathrm{Si}$ ) and the anticorrelations with those depleted in $\mathrm{H}$-burning at high temperature $(\mathrm{O}, \mathrm{Mg})$ are illustrated in Fig. 3.

As a comparison - and as a useful check - we also added to these relations the abundances for 13 RGB stars with highresolution UVES spectra, with $[\mathrm{Al} / \mathrm{Fe}]$ ratios obtained from the classical Al I doublet at 6696-98 $\AA$ in Carretta et al. (2011a). Although none of these 13 giants is in common with the stars in the present sample, the nice agreement we can see in Fig. 3 supports our abundance determination from medium-resolution GIRAFFE spectra.

Using a statistical cluster analysis, we separated two stellar components within NGC 1851 in Carretta et al. (2011a), one more metal-rich/Ba-rich and the other more metal- and Ba-poor, with evidence of a slightly more extreme processing among stars of the second generation of the metal-rich component. In Fig. 4 we show the cumulative distribution of $\mathrm{Al}$ abundance for stars in our present sample divided into the two components: a statistical Kolmogorov-Smirnov test on the cumulative distributions shows evidence that the two distributions are actually different in their $\mathrm{Al}$ abundance, although in this case the metal-rich component seems to be more Al-poor.

The range of $[\mathrm{Al} / \mathrm{Fe}]$ ratios among giants of $\mathrm{NGC} 1851$ is intermediate between the small range observed in GCs like M 4 (e.g. Marino et al. 2008; Carretta et al. 2009b) and the quite wide range observed in massive GCs such as NGC 2808 (Carretta et al. 2009b; Bragaglia et al. 2010).

On the other hand, the Al-Si correlation observed in our GIRAFFE sample (lower right panel in Fig. 3) is found to be statistically significant at a confidence level higher than $97.5 \%$ (Pearson correlation coefficient $r=0.29,57$ degrees of freedom). This correlation demonstrates that part of the material that polluted the gas used in the formation of second generation stars was processed under temperatures higher than about $65 \mathrm{MK}$. Indeed, this is the threshold value above which the reaction ${ }^{27} \mathrm{Al}(\mathrm{p}, \gamma){ }^{28} \mathrm{Si}$ dominates the ${ }^{27} \mathrm{Al}(\mathrm{p}, \alpha){ }^{24} \mathrm{Mg}$ reaction (see Arnould et al. 1999) and a certain amount of ${ }^{28} \mathrm{Si}$ is produced as a leakage from Mg-Al cycle (Karakas \& Lattanzio 2003). The correlation between $\mathrm{Al}$ and $\mathrm{Si}$ (or the corresponding Mg-Si anticorrelation) is now observed in several GCs (see Yong et al. 2005; Carretta et al. 2009b, 2011a) and confirmed to exist also in NGC 1851 by the present large sample.

Finally, we found that the average abundance of Al steadily increases as the chemical composition changes from the pattern 

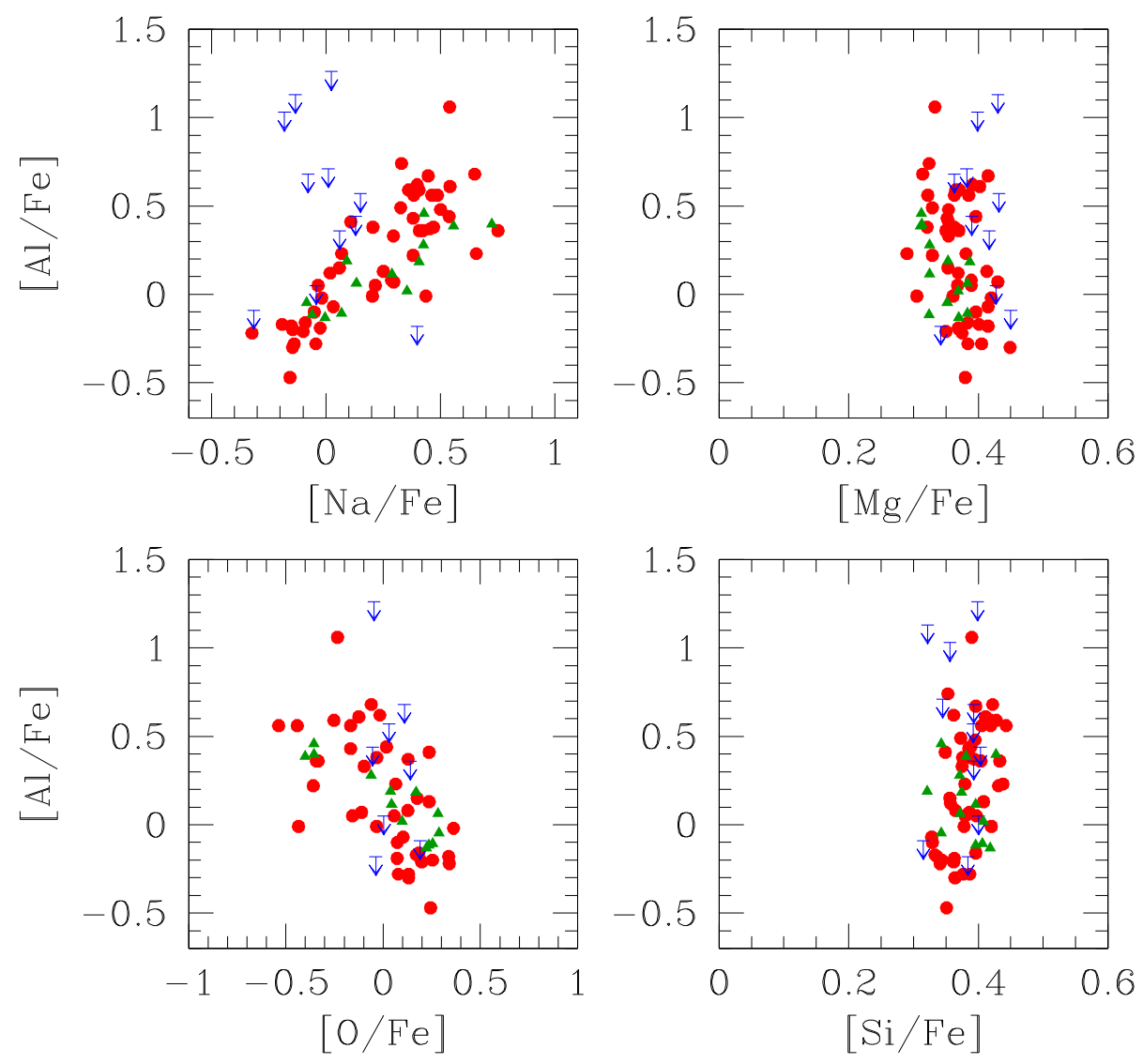

Fig. 3. $[\mathrm{Al} / \mathrm{Fe}]$ ratios derived in this work as a function of abundances of other protoncapture elements from Carretta et al. (2011a): $[\mathrm{Na} / \mathrm{Fe}]$ (upper left panel), $[\mathrm{Mg} / \mathrm{Fe}]$ (upper right), $[\mathrm{O} / \mathrm{Fe}]$ (lower left), and $[\mathrm{Si} / \mathrm{Fe}]$ (lower right). Green triangles are RGB stars with UVES spectra in NGC 1851, from Carretta et al. (2011a). Arrows indicate upper limits in $\mathrm{Al}$ abundances.

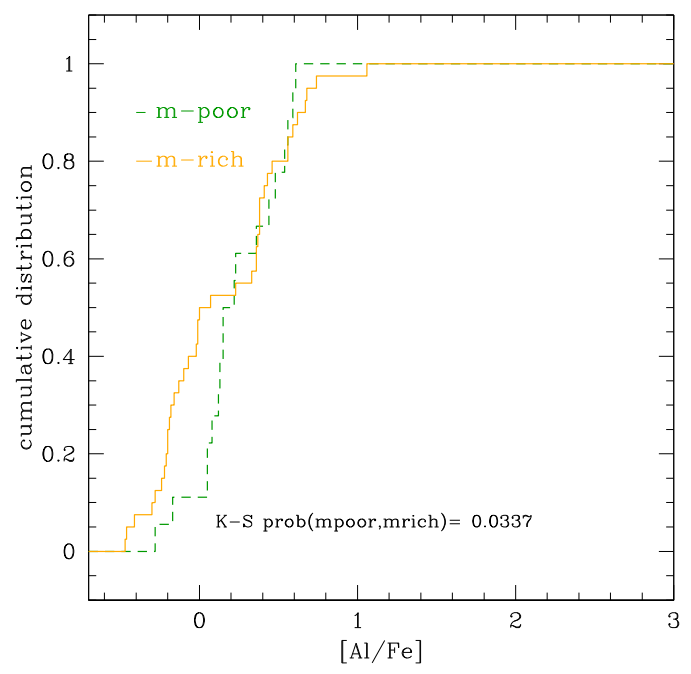

Fig. 4. Cumulative distribution of $[\mathrm{Al} / \mathrm{Fe}]$ ratios from the present work in the metal-poor (green dashed line) and in the metal-rich (orange solid line) component of NGC 1851.

typical of first generation stars to that of second generation stars with increasingly modified composition. By dividing our sample into the three components P, I, and E defined in Carretta et al. (2009a) we found the following average values: $[\mathrm{Al} / \mathrm{Fe}]=$ $-0.11 \pm 0.06 \mathrm{dex}(\mathrm{rms}=0.25,17 \mathrm{stars})$ for the primordial $\mathrm{P}$ component, and $[\mathrm{Al} / \mathrm{Fe}]=+0.36 \pm 0.06 \mathrm{dex}(\mathrm{rms}=0.32,26 \mathrm{stars})$, and $[\mathrm{Al} / \mathrm{Fe}]=+0.56 \pm 0.01 \mathrm{dex}(\mathrm{rms}=0.01,2$ stars $)$ for the $\mathrm{I}$ and $\mathrm{E}$ components, respectively, of second generation stars in NGC 1851. Twelve stars lack O abundances, therefore cannot be classified as P, I or E stars as in Carretta et al. (2009a).

Looking at the relation between proton-capture and neutroncapture elements is particularly interesting in this cluster. First,
Yong \& Grundahl (2008) found a correlation of $\mathrm{Zr}$ and $\mathrm{La}$ with $\mathrm{Al}$ in a few bright giants in NGC 1851. Carretta et al. (2011a) confirmed this correlation using a large sample of more than 120 giants; Gratton et al. (2012b) found a close correlation between $\mathrm{Na}$ and $\mathrm{Ba}$ among $\mathrm{RHB}$ stars; and finally Gratton et al. (2012c) found (i) clearly different $\mathrm{Sr}$ and Ba abundances between the faint (higher abundances) and bright subgiant branches (SGBs) found by Milone et al. (2008); and (ii) that a spread exists within both sequences.

In Fig. 5 we display the ratio $[\mathrm{Al} / \mathrm{Fe}]$ as a function of $[\mathrm{Ba} / \mathrm{Fe}]$ from Carretta et al. (2011a) for stars in the present sample, separating the metal-poor (green triangles) and metal-rich (orange squares) components.

Overall, no correlation is apparent between $\mathrm{Al}$ and $\mathrm{Ba}$; however, when considering the two components separately, there seems to be a correlation among stars of the metal-rich component: the Pearson linear correlation coefficient is 0.29 for a sample of 36 objects, which is significant to a level of confidence higher than $95 \%$. This finding is supported by other evidence, since both $\mathrm{Al}$ (this paper) and Ba (Yong \& Grundahl 2008; Carretta et al. 2011a) are found to be correlated with Na.

Another confirmation comes from the relation of $\mathrm{Al}$ abundances with Strömgren photometry. This set of filters was recently found to be very sensitive to the abundance of light elements such as C and N (see Carretta et al. 2011a,b; Sbordone et al. 2011), hence quite useful when coupled with abundances of light elements to explain segregations or splitting observed in photometric sequences in particular clusters such as NGC 1851.

In Fig. 6 we plot the $y, u-y$ CMD where the upper RGB in NGC 1851 appears well separated. In this CMD stars of our sample are separated according to the $\mathrm{Al}$ abundances: red open symbols and blue filled symbols indicate giants with $[\mathrm{Al} / \mathrm{Fe}]$ higher or lower than the average value of the sample, 0.24 dex. Almost 
E. Carretta et al.: Aluminum abundances in NGC $1851(R N)$

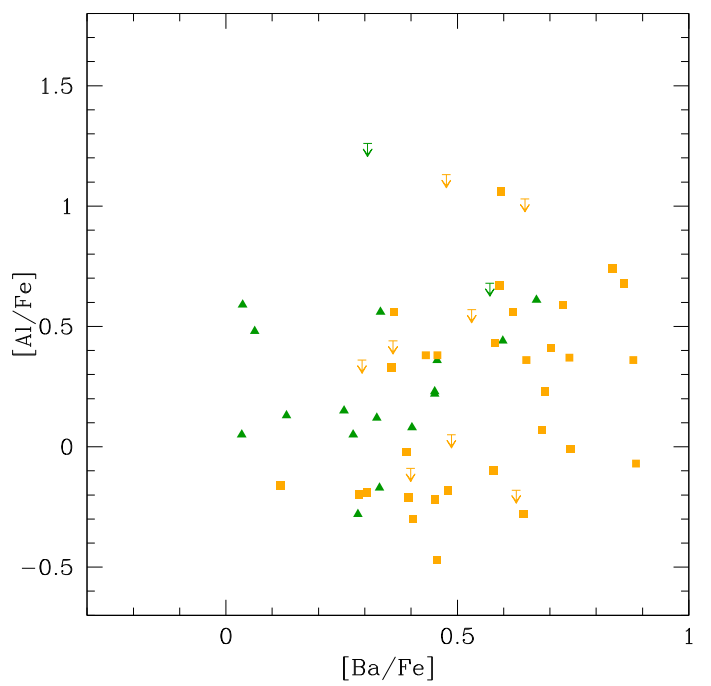

Fig. 5. Run of the $[\mathrm{Al} / \mathrm{Fe}]$ ratio as a function of $[\mathrm{Ba} / \mathrm{Fe}]$ in our sample. Green triangles and orange squares are the metal-poor and metal-rich component, respectively, found by Carretta et al. (2011a) among giants in NGC 1851.

all stars with low Al content (except for three cases) define an extremely narrow stripe on the bluest envelope of the branch, whereas the high-Al stars are more spread out to the red, and the well separated reddest sequence contains almost exclusively high $\mathrm{Al}$ objects, except for the three interlopers with low Al.

In Carretta et al. (2011a,b) we also showed that this red sequence is preferentially populated by giants with high $\mathrm{Ba}$ abundances and that the separation from the bluest sequence is mostly caused by a relevant excess in $\mathrm{N}$.

Taking all these results into account, although this is not a 1 to 1 correlation, the trend of Al-rich stars to occupy preferentially the redder sequence suggests that at least for the upper RGB, the Strömgren colours do measure $\mathrm{N}$ and are not strictly correlated with the progeny of SGB stars, in agreement with what was found in Carretta et al. (2011a) using mainly $\mathrm{O}$ and $\mathrm{Na}$ abundances. In turn, we caution that any identification of the progeny of SGB stars exclusively based on colour (see e.g. Han et al. 2009) must be regarded with caution, because there is no one-to-one correlation, hence these identification may be misleading.

Combined with the results on SGB stars by Gratton et al. (2012c) we can conclude from our study that stars on the faint SGB are predominantly - but non only - N-rich (hence they occupy a redder position on the RGB, in Strömgren colours), while the stars on the bright SGB are about 1/3 N-rich and 2/3 N-poor (hence they are mostly blue in the Strömgren colours).

In summary, the $\mathrm{Al}$ abundances we obtained from spectra acquired as calibration within the Gaia-ESO public survey add another constraint to the complex scenario of the star formation in NGC 1851: the two putative populations hosted in this cluster have not only a spread in $\mathrm{Na}$, but also an $\mathrm{Al}$ spread, and the two are quite similar in both populations.

Acknowledgements. This work was partially funded by the PRIN INAF 2009 grant CRA 1.06.12.10 ("Formation and early evolution of massive star clusters", PI. R. Gratton), and by the PRIN INAF 2011 grant "Multiple populations in globular clusters: their role in the Galaxy assembly" (PI E. Carretta). We thank Sarūnas Mikolaitis for sharing with us the line list for $\mathrm{CN}$ provided by B. Plez. We also thank the referee for her/his constructive suggestions and for asking for a less subjective method, since this resulted in better constrained and accurate abundances. This research has made use of the SIMBAD database (in particular

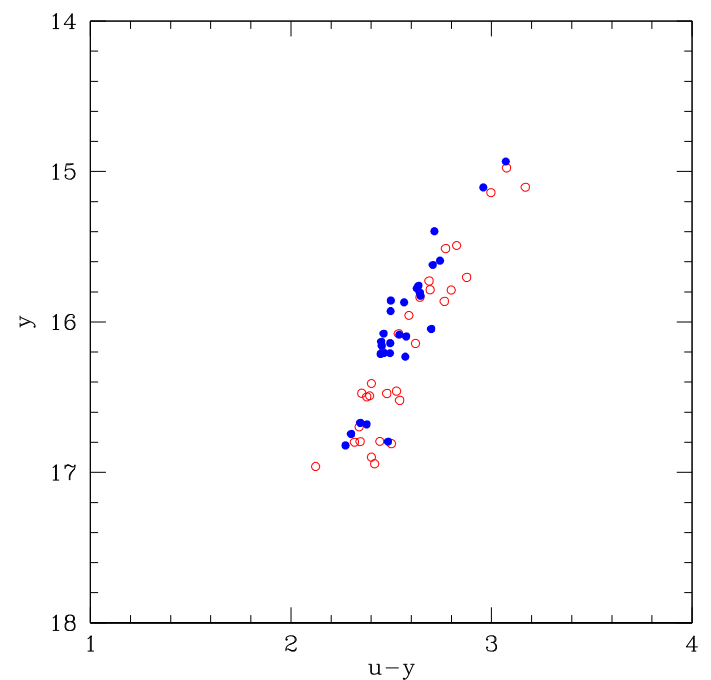

Fig. 6. Strömgren colour-magnitude diagram $y, u-y$ for giants in our sample. Red open and blue filled symbols indicate stars with Al abundances higher and lower than the average ratio $[\mathrm{Al} / \mathrm{Fe}]=0.24$ dex of the sample.

Vizier), operated at CDS, Strasbourg, France and of NASA's Astrophysical Data System.

\section{References}

Arnould, M., Goriely, S., \& Jorissen, A. 1999, A\&A, 347, 572

Bragaglia, A. 2010, IAUS, 268, 119

Bragaglia, A., Carretta, E., Gratton, R. G., et al. 2010, ApJ, 720, L41

Carretta, E. 2006, AJ, 131, 1766

Carretta, E., Bragaglia, A., Gratton, R. G., et al. 2009a, A\&A, 505, 117

Carretta, E., Bragaglia, A., Gratton, R. G., \& Lucatello, S. 2009b, A\&A, 505, 139 (Paper VIII)

Carretta, E., Bragaglia, A., Gratton, R. G., et al. 2010, A\&A, 516, A55

Carretta, E., Lucatello, S., Gratton, R. G., Bragaglia, A., D’Orazi, V. 2011a, A\&A, 533, A69

Carretta, E., Bragaglia, A., Gratton, R. G., D’Orazi, V., \& Lucatello, S. 2011b, A\&A, 535, A121

Carretta, E., Bragaglia, A., Gratton, R. G., Lucatello, S., \& D’Orazi, V. 2012, ApJ, 750, L14

D’Antona, F., \& Caloi, V. 2008, MNRAS, 360, 693

Decressin, T., Meynet, G., Charbonnel, C., Prantzos, N., \& Ekstrom, S. 2007, A\&A, 464, 1029

Denisenkov, P. A., \& Denisenkova, S. N. 1989, A. Tsir., 1538, 11

Gratton, R. G. 1988, Rome Obs. Preprint Ser., 29

Gratton, R. G., Bonifacio, P., Bragaglia, A., et al. 2001, A\&A, 369, 87

Gratton, R. G., Sneden, C., \& Carretta, E. 2004, ARA\&A, 42, 385

Gratton, R. G., Bragaglia, A., Carretta, E., \& Tosi, M. 2006, ApJ, 642, 462

Gratton, R. G., Carretta, E., Bragaglia, A., Lucatello, S., \& D'Orazi, V. 2010, A\&A, 517, A81

Gratton, R. G., Carretta, E., \& Bragaglia, A. 2012a, A\&ARv, 20, 50

Gratton, R. G., Lucatello, S., Carretta, E., et al. 2012b, A\&A, 539, A19

Gratton, R. G., Villanova, S., Lucatello, S., et al. 2012c, A\&A, in press, Doi:10.1051/0004-6361/201219276

Han, S.-I., Lee, Y.-W., Joo, S.-J., et al. 2009, ApJ, 707, L190

Karakas, A. I., \& Lattanzio, J. C. 2003, PASA, 20, 279

Langer, G. E., Hoffman, R., \& Sneden, C. 1993, PASP, 105, 301

Lind, K., Charbonnel, C., Decressin, T., et al. 2011, A\&A, 528, 103

Marino, A. F., Villanova, S., Piotto, G., et al. 2008, A\&A, 490, 625

Marino, A. F., Milone, A., Piotto, G., et al. 2009, A\&A, 505, 1099

Martell, S. L. 2011, Astron. Nachr., 332, 467

Milone, A. P., Bedin, L., Piotto, G., et al. 2008, ApJ, 673, 241

Piotto, G. 2009, in The Ages of Stars, IAU Symp., 258, 233

Sbordone, L., Salaris, M., Weiss, A., \& Cassisi, S. 2011, A\&A, 534, A9

Ventura, P., D’Antona, F., Mazzitelli, I., \& Gratton, R. 2001, ApJ, 550, L65

Yong, D., \& Grundahl, F. 2008, ApJ, 672, L29

Yong, D., Grundahl, F., Nissen, P. E., Jensen, H. R., \& Lambert, D. L. 2005, A\&A, 438, 875 
Table 1. Relevant information and derived abundances for our sample of RGB stars in NGC 1851.

\begin{tabular}{|c|c|c|c|c|c|c|c|c|c|c|c|c|c|}
\hline $\mathrm{Obj}^{1}$ & Star $^{2}$ & $S / N^{3}$ & {$[\mathrm{Al} / \mathrm{Fe}]^{3}$} & err & $\mathrm{Lim}^{3}$ & {$[\mathrm{~N} / \mathrm{Fe}]^{3}$} & $B^{2}$ & $V^{2}$ & {$[\mathrm{Fe} / \mathrm{H}]^{2}$} & {$[\mathrm{O} / \mathrm{Fe}]^{2}$} & {$[\mathrm{Na} / \mathrm{Fe}]^{2}$} & {$[\mathrm{Mg} / \mathrm{Fe}]^{2}$} & {$[\mathrm{Si} / \mathrm{Fe}]^{2}$} \\
\hline 541 & 13618 & 35 & -0.01 & 0.46 & 1 & -0.05 & 16.392 & 15.482 & -1.120 & -0.435 & 0.437 & 0.305 & 0.420 \\
\hline 1461 & 14827 & 32 & 0.74 & 0.36 & 1 & & 17.819 & 16.994 & -1.164 & & 0.330 & 0.324 & 0.353 \\
\hline 974 & 16120 & 98 & 0.41 & 0.09 & 1 & 0.10 & 16.859 & 16.012 & -1.103 & 0.236 & 0.109 & 0.354 & 0.349 \\
\hline 1218 & 20189 & 34 & 0.44 & & 0 & 0.70 & 17.339 & 16.521 & -1.155 & -0.055 & 0.129 & 0.390 & 0.403 \\
\hline 1186 & 20426 & 38 & 0.36 & & 0 & 0.30 & 17.281 & 16.429 & -1.106 & 0.139 & 0.060 & 0.417 & 0.393 \\
\hline 1044 & 20653 & 35 & 0.08 & 0.28 & 1 & 0.55 & 17.002 & 16.120 & -1.184 & 0.127 & 0.287 & 0.389 & 0.365 \\
\hline 733 & 20922 & 41 & 0.05 & & 0 & 0.10 & 16.705 & 15.784 & -1.142 & 0.004 & -0.043 & 0.427 & 0.400 \\
\hline 766 & 21453 & 60 & 0.05 & 0.15 & 1 & 0.25 & 16.746 & 15.835 & -1.156 & -0.157 & 0.216 & 0.368 & 0.380 \\
\hline 1211 & 21830 & 45 & 0.38 & 0.22 & 1 & 0.50 & 17.344 & 16.515 & -1.163 & -0.033 & 0.470 & 0.363 & 0.388 \\
\hline 1103 & 22360 & 60 & -0.09 & & 0 & 0.10 & 17.123 & 16.223 & -1.145 & 0.191 & -0.315 & 0.450 & 0.315 \\
\hline 788 & 22588 & 80 & -0.28 & 0.23 & 1 & 0.10 & 16.762 & 15.878 & -1.200 & 0.078 & -0.139 & 0.405 & 0.377 \\
\hline 891 & 22813 & 120 & -0.30 & 0.13 & 1 & 0.20 & 16.820 & 15.945 & -1.101 & 0.131 & -0.146 & 0.449 & 0.364 \\
\hline 719 & 23647 & 57 & 0.22 & 0.16 & 1 & 0.00 & 16.693 & 15.806 & -1.193 & -0.359 & 0.381 & 0.329 & 0.431 \\
\hline 810 & 23765 & 61 & 0.36 & 0.14 & 1 & 0.05 & 16.765 & 15.814 & -1.145 & -0.344 & 0.409 & 0.370 & 0.403 \\
\hline 1016 & 25037 & 63 & -0.28 & 0.29 & 1 & 0.30 & 16.975 & 16.106 & -1.151 & 0.131 & -0.044 & 0.384 & 0.387 \\
\hline 1202 & 25799 & 38 & 0.68 & & 0 & & 17.344 & 16.493 & -1.214 & 0.109 & -0.078 & 0.363 & 0.393 \\
\hline 1190 & 26532 & 41 & 0.68 & 0.24 & 1 & & 17.293 & 16.472 & -1.180 & -0.061 & 0.650 & 0.314 & 0.422 \\
\hline 1045 & 26552 & 57 & -0.18 & & 0 & 0.10 & 17.019 & 16.090 & -1.114 & -0.038 & 0.399 & 0.342 & 0.384 \\
\hline 1291 & 26880 & 57 & -0.18 & 0.33 & 1 & 0.30 & 17.537 & 16.699 & -1.161 & 0.337 & -0.151 & 0.415 & 0.336 \\
\hline 1274 & 27491 & 43 & -0.01 & 0.48 & 1 & 0.40 & 17.499 & 16.685 & -1.177 & -0.034 & 0.203 & 0.361 & 0.378 \\
\hline 1070 & 28116 & 32 & 0.71 & & 0 & 0.40 & 17.040 & 16.200 & -1.189 & & 0.010 & 0.382 & 0.345 \\
\hline 418 & 29203 & 57 & 0.56 & 0.14 & 1 & 0.10 & 16.162 & 15.115 & -1.157 & -0.442 & 0.487 & 0.363 & 0.443 \\
\hline 1232 & 29470 & 41 & 0.59 & 0.25 & 1 & 0.40 & 17.413 & 16.548 & -1.144 & & 0.361 & 0.371 & 0.428 \\
\hline 1024 & 30286 & 28 & 0.57 & & 0 & 0.20 & 16.985 & 16.115 & -1.072 & 0.031 & 0.151 & 0.432 & 0.392 \\
\hline 802 & 31284 & 75 & 0.05 & 0.12 & 1 & 0.00 & 16.767 & 15.893 & -1.179 & 0.057 & -0.034 & 0.389 & 0.397 \\
\hline 1307 & 31399 & 48 & -0.02 & 0.38 & 1 & & 17.569 & 16.761 & -1.158 & 0.362 & -0.018 & 0.420 & \\
\hline 1410 & 31463 & 30 & 1.13 & & 0 & & 17.774 & 17.031 & -1.163 & & -0.134 & 0.430 & 0.322 \\
\hline 1046 & 31520 & 61 & 0.44 & 0.16 & 1 & 0.00 & 16.998 & 16.158 & -1.230 & 0.017 & 0.539 & 0.396 & 0.388 \\
\hline 553 & 32112 & 43 & 0.43 & 0.21 & 1 & 0.30 & 16.460 & 15.517 & -1.077 & -0.167 & 0.381 & 0.352 & 0.385 \\
\hline 1012 & 32256 & 44 & -0.10 & 0.38 & 1 & & 16.947 & 16.109 & -1.168 & 0.073 & -0.051 & 0.396 & 0.329 \\
\hline 875 & 35750 & 66 & 0.36 & 0.13 & 1 & 0.20 & 16.813 & 15.874 & -1.185 & -0.334 & 0.423 & 0.356 & 0.404 \\
\hline 347 & 35999 & 49 & 1.06 & 0.18 & 1 & 0.00 & 15.962 & 14.971 & -1.147 & -0.235 & 0.541 & 0.333 & 0.390 \\
\hline 1395 & 36292 & 27 & 0.37 & 0.33 & 1 & & 17.724 & 16.917 & -1.101 & 0.128 & 0.454 & 0.365 & 0.394 \\
\hline 757 & 38484 & 29 & 0.61 & 0.30 & 1 & & 16.727 & 15.837 & -1.198 & -0.125 & 0.542 & 0.402 & 0.411 \\
\hline 1324 & 38818 & 40 & 0.23 & 0.24 & 1 & 0.00 & 17.618 & 16.804 & -1.144 & & 0.657 & 0.290 & 0.438 \\
\hline 801 & 39364 & 54 & 0.33 & 0.16 & 1 & 0.20 & 16.765 & 15.877 & -1.128 & -0.098 & 0.295 & 0.354 & 0.375 \\
\hline 539 & 40300 & 124 & 0.49 & 0.07 & 1 & -0.10 & 16.414 & 15.533 & -1.192 & & 0.327 & 0.329 & 0.373 \\
\hline 409 & 40615 & 72 & 0.59 & 0.12 & 1 & -0.20 & 16.114 & 15.156 & -1.231 & -0.253 & 0.407 & 0.365 & 0.419 \\
\hline 692 & 41113 & 68 & 0.56 & 0.14 & 1 & 0.20 & 16.642 & 15.769 & -1.219 & -0.537 & 0.463 & 0.322 & 0.419 \\
\hline 1323 & 41855 & 35 & 0.48 & 0.30 & 1 & & 17.610 & 16.847 & -1.202 & & 0.501 & 0.354 & 0.395 \\
\hline 1339 & 43528 & 41 & -0.22 & 0.45 & 1 & 0.40 & 17.624 & 16.854 & -1.067 & 0.340 & -0.323 & 0.375 & 0.341 \\
\hline 1351 & 44224 & 38 & 0.36 & 0.27 & 1 & & 17.660 & 16.839 & -1.205 & & 0.752 & 0.350 & 0.433 \\
\hline 616 & 44414 & 56 & 0.23 & 0.18 & 1 & 0.10 & 16.549 & 15.653 & -1.187 & 0.064 & 0.069 & 0.381 & 0.379 \\
\hline 346 & 44939 & 147 & -0.19 & 0.10 & 1 & -0.20 & 15.952 & 14.956 & -1.151 & 0.072 & -0.025 & 0.369 & 0.363 \\
\hline 1119 & 45006 & 63 & -0.07 & 0.28 & 1 & 0.00 & 17.096 & 16.245 & -1.115 & 0.103 & 0.032 & 0.415 & 0.328 \\
\hline 1080 & 45090 & 59 & 0.15 & 0.16 & 1 & & 17.047 & 16.228 & -1.185 & 0.175 & 0.059 & 0.353 & 0.356 \\
\hline 1050 & 45413 & 88 & -0.47 & 0.21 & 1 & 0.30 & 16.981 & 16.155 & -1.125 & 0.244 & -0.157 & 0.380 & 0.351 \\
\hline 1093 & 46228 & 73 & -0.21 & 0.21 & 1 & & 17.075 & 16.238 & -1.097 & 0.198 & -0.099 & 0.350 & 0.362 \\
\hline 1203 & 46657 & 30 & 1.26 & & 0 & & 17.316 & 16.521 & -1.246 & -0.047 & 0.023 & & 0.399 \\
\hline 1074 & 46958 & 93 & -0.20 & 0.20 & 1 & 0.25 & 17.048 & 16.204 & -1.142 & 0.254 & -0.145 & 0.371 & 0.344 \\
\hline 507 & 47385 & 66 & -0.17 & 0.27 & 1 & 0.00 & 16.341 & 15.437 & -1.203 & 0.171 & -0.191 & 0.401 & 0.333 \\
\hline 746 & 47795 & 38 & 0.56 & 0.23 & 1 & 0.40 & 16.723 & 15.753 & -1.127 & -0.167 & 0.384 & 0.385 & 0.405 \\
\hline 407 & 48085 & 119 & -0.16 & 0.11 & 1 & -0.20 & 16.115 & 15.144 & -1.111 & 0.183 & -0.090 & 0.384 & 0.396 \\
\hline 1350 & 48277 & 26 & 1.03 & & 0 & & 17.659 & 16.863 & -1.068 & & -0.181 & 0.399 & 0.356 \\
\hline 1007 & 48388 & 58 & 0.12 & 0.16 & 1 & 0.00 & 16.937 & 16.136 & -1.163 & & 0.018 & 0.369 & 0.357 \\
\hline 1144 & 49965 & 45 & 0.62 & 0.20 & 1 & 0.30 & 17.156 & 16.242 & -1.095 & -0.017 & 0.399 & 0.391 & 0.362 \\
\hline 1280 & 50876 & 37 & 0.38 & 0.23 & 1 & 0.30 & 17.512 & 16.680 & -1.097 & & 0.205 & 0.321 & 0.376 \\
\hline 588 & 50973 & 64 & 0.07 & 0.14 & 1 & -0.20 & 16.530 & 15.572 & -1.084 & -0.111 & 0.296 & 0.430 & 0.386 \\
\hline 738 & 51311 & 62 & 0.13 & 0.16 & 1 & 0.30 & 16.706 & 15.758 & -1.256 & 0.236 & 0.251 & 0.413 & 0.408 \\
\hline 1319 & 52579 & 33 & 0.67 & 0.28 & 1 & & 17.587 & 16.801 & -1.151 & & 0.447 & 0.415 & 0.396 \\
\hline
\end{tabular}

Notes. ${ }^{(1)}$ Object identification, from FLAMES mask in the ESO archive. ${ }^{(2)}$ Star identification, magnitudes and abundance ratios taken from Carretta et al. (2011). ${ }^{(3)} \mathrm{S} / \mathrm{N}$ and abundances derived in the present work. Lim is 0 for upper limits in the $\mathrm{Al}$ abundance. 